\title{
A meta-analysis of serological thymidine kinase 1 as a marker for colorectal benign and malignant tumor risk assessment
}

\author{
LI DANG ${ }^{*}$, HONGBO MA* , AILIAN HEI, SHUAI XU, JI ZHOU, ELLEN HE and SVEN SKOG \\ Department of Medicine, Shenzhen Sino-Swed Precision Medicine Institute, \\ Shenzhen, Guangdong 518057, P.R. China
}

Received May 31, 2019; Accepted October 10, 2019

DOI: $10.3892 /$ mco.2020.2002

\begin{abstract}
The present study investigated whether a concentration of serum thymidine kinase 1 (STK1p) could be used to distinguish between healthy individuals, patients with colorectal benign tumors and individuals with colorectal cancer (CRC). The effectiveness of surgery on patients with CRC was monitored. A total of 20 publications containing patients with $\mathrm{CRC}(\mathrm{n}=1,836)$, patients with colorectal benign tumors $(n=774)$ and healthy controls $(n=1,701)$ were analysed in the present meta-analysis. The publications were collected from PubMed, Embase, CENTRAL, CNKI, Wanfang, VIP and SinoMed databases from January 1, 2009 until August 31, 2019. Articles were analyzed according to sensitivity (Forest plot) and publication bias (Begg's plot, Egger's linear regression) using fixed or random effect models to calculate the weighted mean difference. Study quality was checked using the Newcastle-Ottawa Scale Document Quality Assessment Scale. The meta-analysis followed the PRISMA statement. The results revealed that STK1p significantly distinguished healthy individuals and those with colorectal benign tumors from patients with CRC, and from patients with benign tumors $(\mathrm{P}<0.000001)$. STK1p levels also decreased by $40 \%$ following surgery $(\mathrm{P}<0.0001)$, which corresponded to half-life of $\sim 1$ month. The quality of the present study was high and no bias was identified among publication. It was concluded that STK1p was a reliable biomarker for the early detection of benign lesions, which may therefore prevent their future development into colorectal malignancies. STK1p may also be used for the clinical dynamic monitoring of the effectiveness of surgery in patients with CRC. Combining
\end{abstract}

Correspondence to: Professor Sven Skog, Department of Medicine, Shenzhen Sino-Swed Precision Medicine Institute, 1st Goaxin C. Avenue, Kexing Science Park, Shenzhen, Guangdong 518057, P.R. China

E-mail: svenisak@icloud.com

*Contributed equally

Key words: thymidine kinase 1, serum thymidine kinase 1 concentration, colorectal cancer, neoplastic colorectal polyp (colorectal benign tumor), meta-analysis
STK1p with colorectal-associated biomarkers, in addition to the determination of tumor stage and grade may therefore be of use.

\section{Introduction}

According to the World Health Organization, the number of newly diagnosed colorectal cancer (CRC) cases will increase by $77 \%$ and the number of deaths by $80 \%$ by $2030(1,2)$. The expected increase in CRC will mainly occur in less developed countries, due to the development of a lifestyle closed to Western countries (3-5).

The neoplastic transformation time of CRC is 10-15 years (6-9), and the 5 year survival following treatment $50-60 \%(10,11)$. Reliable diagnostic, prognostic and predictive biomarkers are required (12). At present, the most accepted tests for CRC are fecal occult blood test, colonoscopy and sigmoidoscopy (13). Tumor node metastasis (TNM) staging and carcinoembryonic antigen level are also used. However, most of these tests have been reported to be too insensitive for accurate individual prognosis $(14,15)$. Tumor biomarkers with a high sensitivity and specificity are required. In this meta-analysis, the use of serum thymidine kinase 1 (STK1p) concentration for prognosis and treatment monitoring in CRC patients is discussed.

STK1p has been proven useful for predicting recurrence and survival in many types of human cancer $(16,17)$. TK1 is a kinase enzyme that converts deoxythymidine to deoxythymidine monophosphate and is involved in the synthesis of DNA, and thus related to cell growth rate (proliferation). The new-generation STK1p concentration assay shows an area under the curve (AUC) value of 0.96 (18), and is a more reliable assay than the serum TK activity $(19,20)$ and serum TK1 sandwich ELISA (21) assays. Low STK1p values are associated with a better prognosis $(16,18,22-27)$. The STK1p marker is an independent prognostic factor for survival in CRC patients $(n=504)(28)$. STK1p can distinguish malignant patients from benign tumor patients and healthy individuals, as well as predict the prognosis of survival and relapse. STK1p can also monitor the effect of the treatment, and is a useful biomarker for predicting the development of malignancies and discovering early-stage tumors $(18,26,28)$.

A number of studies have focused on the use of STK1p in colorectal benign and malignant tumor patients, but most of them included a limited number of cases, thus reducing the reliability of the conclusions. Therefore, 20 colorectal studies 
were collected for the present meta-analysis, in order to obtain a sufficient number of cases. The results showed that STK1p values are significantly higher in CRC patients, as compared to heathy individuals or patients with benign tumors. STK1p was also used to monitor the effect of the surgery.

\section{Materials and methods}

Literature search. A systematic literature search was conducted through the PubMed, Embase, CENTRAL, CNKI, Wanfang, VIP and SinoMed databases until August 31, 2019, using the following strategy keywords: ('thymidine kinase 1' or 'TK1') and ('colorectal' or 'colon' or 'rectal' or 'colorectum' or 'rectum') and ('cancer' or 'tumor' or 'carcinoma' or 'malignancy'). A more detailed description of the search strategy used is described in the beginning of the Results section. The literature search was restricted to human studies. There were no language restrictions.

The meta-analysis followed the PRISMA guidelines. The quality of the meta-analysis as a study was investigated using the Newcastle-Ottawa Scale Document Quality Assessment Scale (NOS). Only studies using the STK1p assay developed by us, tested in many clinical studies and statistically proved to be reliable were selected (summary in refs. 27,28), in order to guarantee the high quality of the STK1p results. Since no specific review protocol suitable for our meta-analysis was found, our own protocol was used.

All analyses were based on previously published studies, and therefore no ethical approval and patient consent were required.

\section{Inclusion and exclusion criteria}

Inclusion criteria. i) STK1p as an endpoint; ii) STK1p was measured by the kit developed by SSTK Ltd; iii) patients with adenomatous polyps were identified by clinical endoscopy and pathological diagnosis. Patients were identified by pathological diagnosis, classified as stage I-III and grade 1-3, and confirmed to have no residual tumor following surgery; iv) healthy individuals were used as the control group. Healthy people were defined as disease-free checked by different imaging, blood tests and other pathological methods.

Exclusion criteria. i) Insufficient data; ii) TK1 immunohistochemistry and TK1 activity; iii) Invalid research data, which included physiological stress responses such as immunological reaction, inflammation and activation of metabolic adenosine mediators. Oxidative stress was considered a surgical stress response, together with myocardial injury, sepsis, pulmonary edema, and kidney and liver failure, which could increase mortality; iv) Healthy people were excluded when containing diseases associated with tumors proliferation, such as precancerous (moderate/severe types of hyperplasia of breast, prostate, gastrointestinal, cervix, liver cirrhosis, refractory anemia). Also excluded were people with risk-diseases associated with tumors progression such as liver disease, moderate/severe fatty liver, high risk for hepatitis B, abnormal liver function, obesity and benign tumors (such as renal, thyroid); and any of the following conditions: severe cardiac disease; using any medication that could affect the STK1 levels such as exogenous hormone therapy; pregnancy; or acute illness such as inflammation/virus infection within 4 weeks.
Table I. No of publications in the group of controls, benign and malignant persons, no of samples and STK1p mean values in the various groups included in the present study.

\begin{tabular}{lccc}
\hline Variables & Control & Benign & Malignant \\
\hline Publications (n) & 19 & 10 & 20 \\
Samples (n) & 1,701 & 774 & 1,836 \\
STK1p (mean \pm SD) & $0.88 \pm 0.50$ & $1.30 \pm 0.84$ & $3.14 \pm 2.55$ \\
P-value & $0.00078^{\mathrm{a}}$ & $<0.0001^{\mathrm{b}}$ & $<0.0001^{\mathrm{c}}$ \\
\hline
\end{tabular}

P-values corresponding to the comparison of STK1p values between groups. ${ }^{a}$ Control vs. benigh group, benigh vs. malignant group and ${ }^{c}$ malignant vs. control group. SD, standard deviation; STK1p serum thymidine kinase 1 .

\section{Literature screening and data extraction}

Primary screening. The title and abstract of literatures were carefully reviewed, and $10 \%$ of the excluded papers were randomly selected to check the concordance rate.

Secondary screening. After checking the abstracts, the full text of the papers was re-evaluated, and it was decided whether these papers should be included to the study or not, according to the criteria. Authors 1, 2 and 3 screened papers independently and discussed to reach an agreement; when met with a disagreement, the papers were rechecked by authors 5 and 6 .

The following data were extracted from each study: First author's name, publication year, title of publication, published journal, study population, number of samples, design type and results.

Statistical analysis. RevMan 5.1 statistical software provided by Networks of Cochrane Review Groups and Stata 12.0 data analysis and statistical software were used (29). A heterogeneity test was performed at the beginning, and depending on the results, a fixed or random effects model was used. A fixed effects model with an $\mathrm{I}^{2}$ of $<50 \%$ or random effects model with an $\mathrm{I}^{2}$ of $>50 \%$ was used to calculate the weighted mean difference and $95 \%$ confidence interval. In addition, Funnel plot and Egger's linear regression test was used to assess literature bias. For the comparison of STK1p concentration among the different groups of controls and patients, one-way analysis of variance followed by a post hoc least significant difference test was performed. SPSS version 19 was utilized for statistical analysis (IBM Corp.). $\mathrm{P}<0.05$ was considered to indicate a statistically significant difference.

\section{Results}

Literature search and study characteristics. The healthy control group included individuals with no evidence of tumors. Data from patients with colorectal neoplastic (adenomatous) polyps were used in the meta-analysis. Adenomatous polyps are benign tumors that originate from the mucus-secreting colonic epithelial cells (9). Tumors from CRC patients pathologically identified as clinical I-III degree and grade G1-G3 were defined as malignant. We had no information on whether 


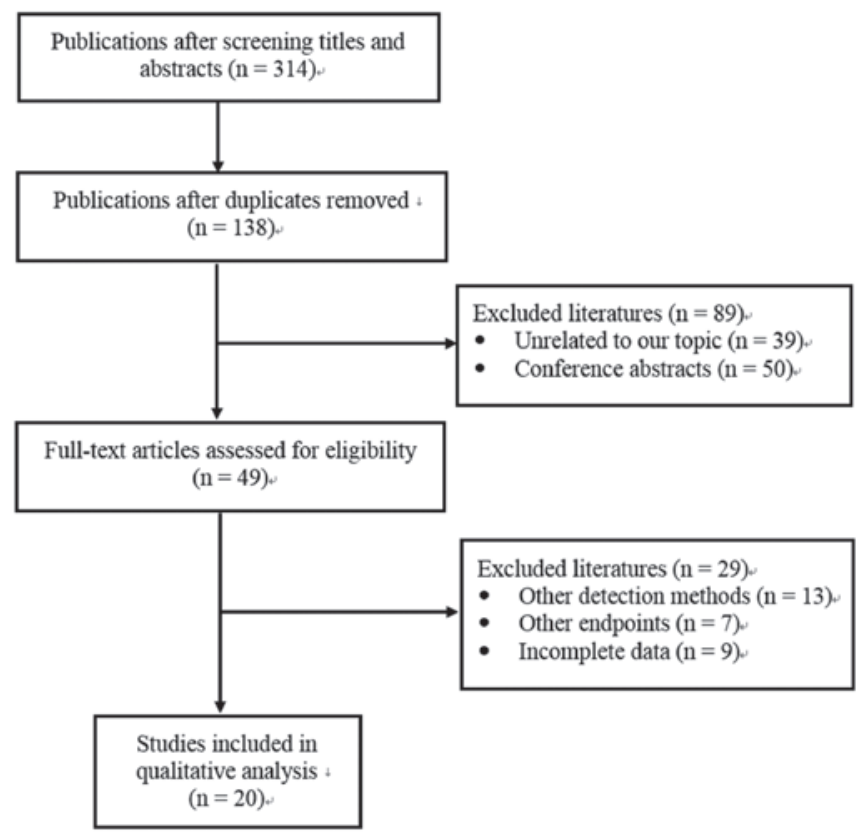

Figure 1. Flow diagram of the literature search and study selection process.

other types of tumors besides CRC were identified in the benign or malignant tumor groups.

As shown in Fig. 1, a total of 314 publications were initially identified through a search of the Pubmed, Embase, CENTRAL, CNKI, Wanfang, VIP and SinoMed databases. A total 176 publications were removed due to duplications and 138 were kept. Next, 89 publications were excluded from the remaining articles following a screening of the titles and abstracts, due to being conference abstracts or focusing on unrelated topics. Therefore, 49 full-text articles were assessed for eligibility. Among them, 13 were excluded, since another STK1 method was used instead of the SSTK dot blot ECL assay, 7 because they used different endpoints, and 9 due to incomplete data. Finally, 20 studies were included in this meta-analysis (30-49).

The number of manuscripts, patients involved and the STK1p mean values in the control, benign and malignant tumor groups are shown in Table I. Detailed information on the number of cases, age distribution, type of CRC, clinical stages grades and just before start of the surgery and one month after surgery reported in the individual publications are presented in Table II.

A total of 1,836 CRC patients were included in the 20 publications, including 774 benign tumor patients and 1,701 healthy individuals. The majority of CRC patients were male with an age-distribution of 16-85 years and colon cancer clinical stage of III and grade low/medium/high. The expression of STK1p increased significantly in the following manner: Controls < benign $<$ malign $(\mathrm{P}<0.001)$. The quality of the meta-analysis as a study was checked by the NOS and was found to be high, with 19 publications meeting 8 of the requirements of NOS, and 1 publication meeting 6 . The results are presented in Table III.

STK1p values in different populations. The STK1p values were significantly different between healthy individuals and benign tumor patients, healthy individuals and CRC patients, and benign tumor and CRC patients (Table I, Fig. 2A). The number of publications involved among the healthy controls were 19 , the benign 10 and the malignant 20, corresponding to 1,701, 774 and 1,836 persons, respectively. The STK1p values decreased significantly (40\%) 1 month after surgery (Fig. 2B).

Meta-analysis statistical calculation. Significant values were calculated using statistical programs for meta-analysis (see Material and methods sections). The results for the different test groups are shown in Figs. 3 and 4. The statistical values were calculated between healthy individuals and benign tumor patients (Fig. 3B), healthy individuals and CRC malignant tumor patients (Fig. 3A), benign and malignant tumor patients (Fig. 3C), and before and after surgery (Fig. 3D).

STK1p of healthy individuals compared to benign tumor patients. In this statistical calculation 1,052 healthy individuals and 671 benign tumor patients from 9 studies were used. Based on a random effects model, the heterogeneity test showed that the STK1p value in healthy individuals was significantly lower than that in benign tumor patients (Fig. 3B).

STK1p of healthy individuals compared to CRC patients. Of the 20 publications received, 19 were used in this comparison, including 1,701 healthy individuals and 1,703 patients with CRC (Fig. 3A). A heterogeneity test based on the random effects model showed that the STK1p value in CRC patients was statistically higher than that in healthy individuals.

STK1p in benign tumor patients compared to CRC patients. Out of the 20 publications, 10 included data regarding STK1p in benign tumors and CRC (Fig. 3C). There was a total of 774 cases in the benign tumor group and 904 in the CRC group. A heterogeneity test based on the random effects model showed that the STK1p values were significantly higher in CRC patients, as compared to benign tumor patients. 


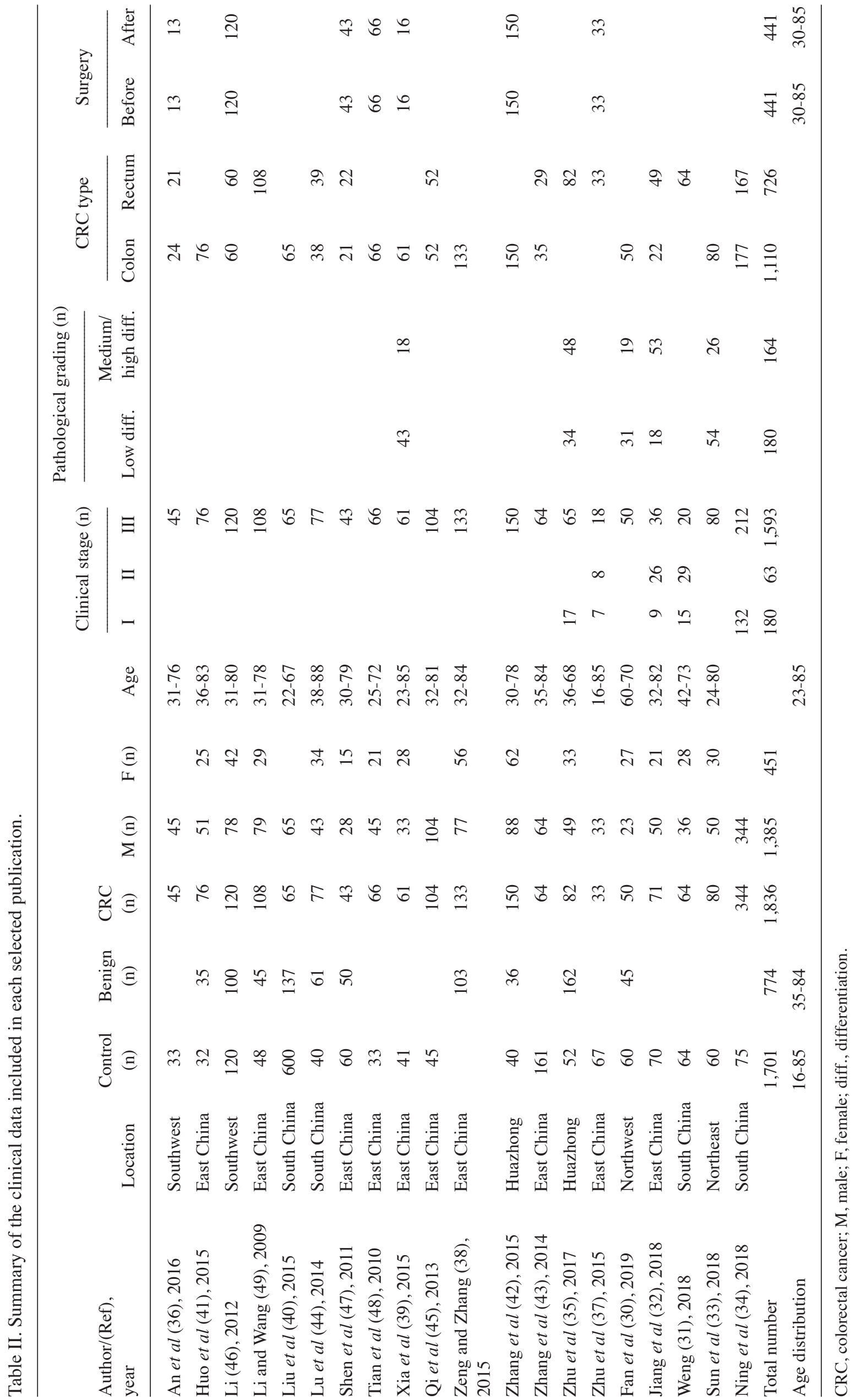




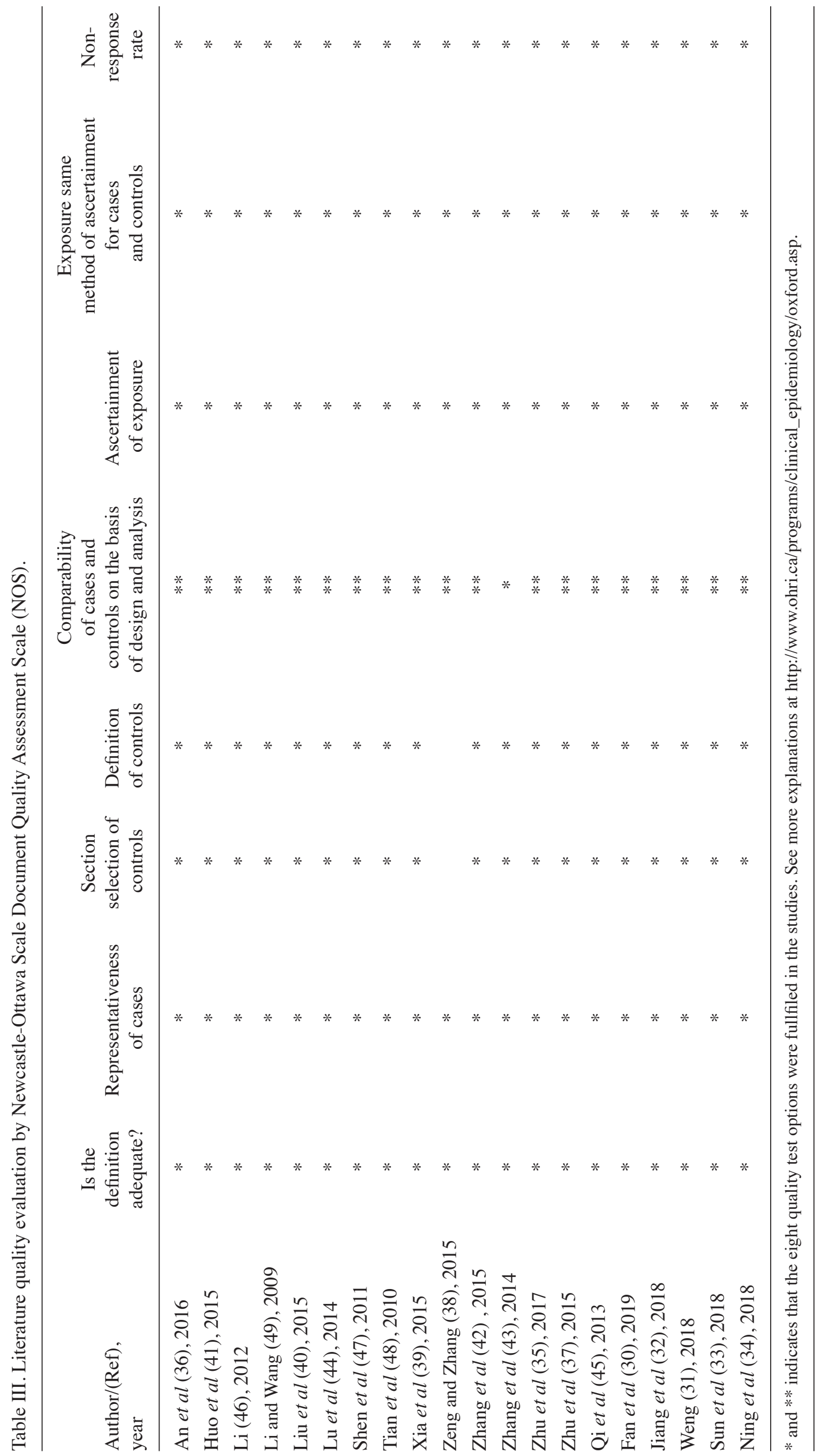


Table IV. Egger's linear regression test for the assessment of publication bias.

\begin{tabular}{|c|c|c|c|c|c|}
\hline Standard effect & Coefficient & Standard error & $\mathrm{T}$ value & $\mathrm{p}>|\mathrm{t}|$ & $95 \% \mathrm{CI}$ \\
\hline \multicolumn{6}{|c|}{ Control vs. benign } \\
\hline Slope & -0.8601885 & 0.2631424 & -3.27 & 0.014 & -1.482421 to -0.2379556 \\
\hline Bias & 4.134483 & 2.616981 & 1.58 & 0.158 & -2.053694 to 10.32266 \\
\hline \multicolumn{6}{|c|}{ Control vs. malignant } \\
\hline Slope & -2.533914 & 0.249529 & -10.15 & 0.000 & -3.060375 to -2.007454 \\
\hline Bias & 1.303459 & 2.242794 & 0.58 & 0.569 & -3.428422 to 6.03534 \\
\hline \multicolumn{6}{|c|}{ Benign vs. malignant } \\
\hline Slope & -2.50849 & 0.6528756 & -3.84 & 0.005 & -4.014024 to -1.002956 \\
\hline Bias & 5.555459 & 4.287575 & 1.30 & 0.231 & -4.331706 to 15.44262 \\
\hline \multicolumn{6}{|c|}{ Before vs. after treatment } \\
\hline Slope & 1.414582 & 0.2186961 & 6.47 & 0.001 & 0.8524054 to 1.976758 \\
\hline Bias & -0.6030739 & 1.460007 & -0.34 & 0.744 & -4.256143 to 3.249995 \\
\hline
\end{tabular}

CI, confidence interval.
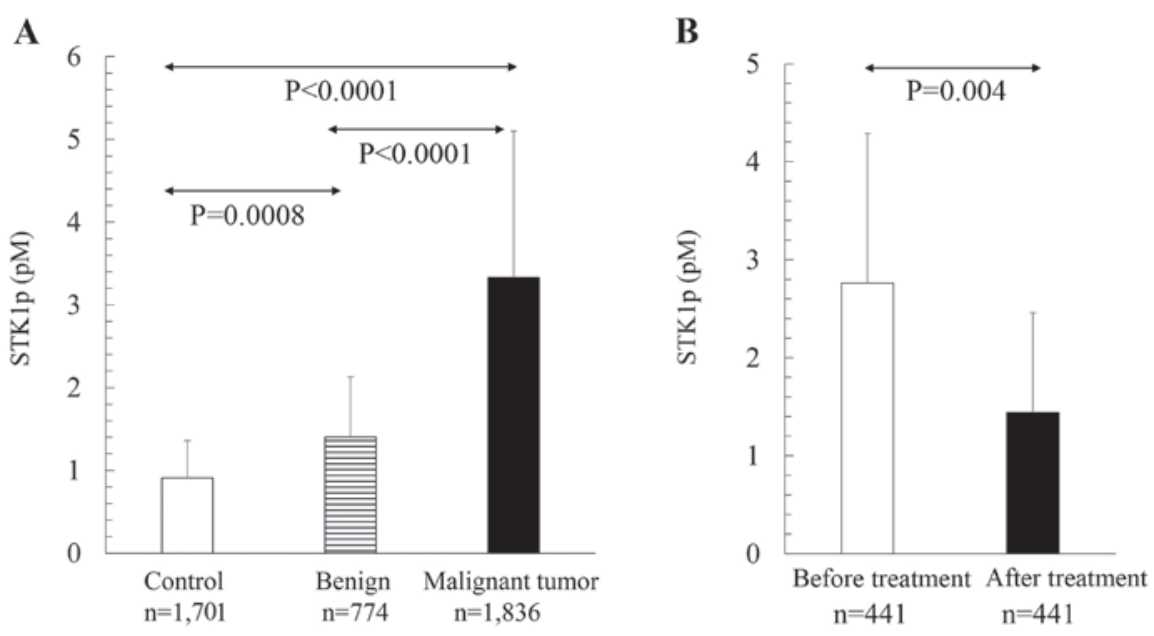

Figure 2. STK1p levels. (A) STK1p levels in healthy controls, benign patients and patients with malignant colorectal cancer patients. (B) STK1p levels in patients with malignant colorectal cancer before and after treatment. Data are presented as the mean \pm standard deviation and significant values were determined using a t-test. STK1p, serum thymidine kinase 1.

STK1p level before and after surgery. All 20 publications included data regarding the STK1p level before and 1 month after surgery. A heterogeneity test, based on the random effects model, showed a significant decrease by $40 \% 1$ month after surgery (Fig. 3D).

Sensitivity analysis. Sensitivity analysis was conducted to evaluate the effect of excluding any individual study. By excluding 1 article at a time in turn, the summary results of the remaining literatures did not substantially change (Fig. 4A).

Publication bias. Begg's Funnel-plot (Fig. 4B) and Egger' test (Table IV) were used to examine the potential publication bias. There was no evidence of bias between the healthy individuals and the benign tumor group, healthy individuals and the malignant group, the benign and malignant tumor groups, or the before and after treatment groups. The Egger's test showed that the possibility of potential bias from each comparison analysis was very low (all P>0.05; Table IV). Based on these results, no significant publication bias was identified in this meta-analysis.

\section{Discussion}

Meta-analysis is a statistical analysis that combines the results of multiple studies (50-52). In the present study, a meta-analysis was performed according to the aforementioned inclusion and exclusion criteria. No bias was found. We also found that STK1p was able to distinguish between healthy individuals and benign tumor patients, as well as between healthy/benign tumor and malignant tumor patients. STK1p also monitored the results of the surgery. Thus, STK1p is a reliable biomarker for the prognosis of benign tumor and CRC patients, and a useful follow-up tool for surgery.

The STK1p was determined by an assay with a high sensitivity (0.80) and specificity (0.99), and an AUC value of 0.96 . This assay is the most sensitive assay for TK1 in the serum on 
A

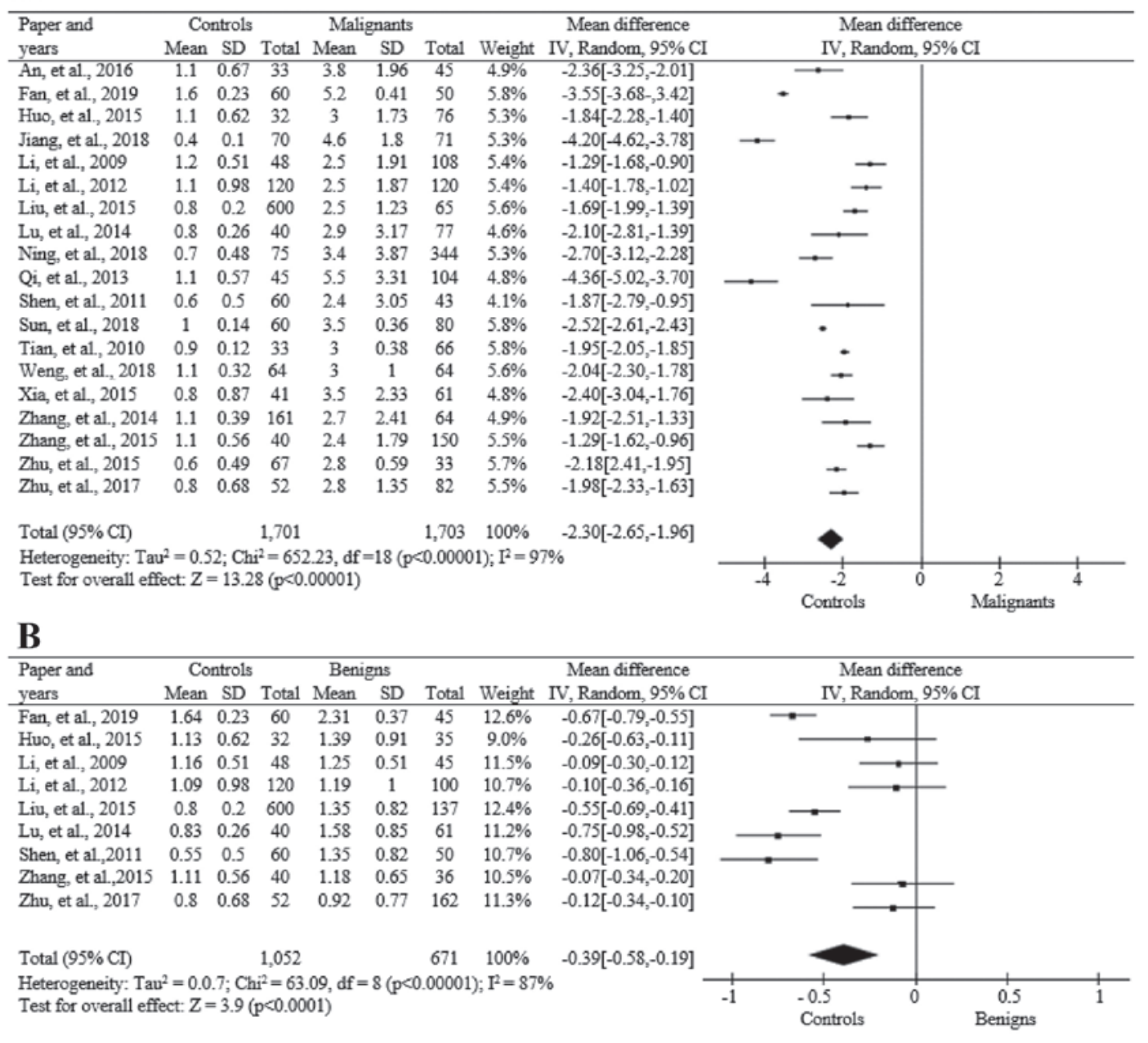

C

\begin{tabular}{lccccccccc|c}
\hline $\begin{array}{l}\text { Paper and } \\
\text { years }\end{array}$ & Mean & SD & Total & Mean & SD & Total Weight & Mean difference Random, $95 \%$ CI & & Mean difference \\
IV, Random, 95\% CI
\end{tabular}

D

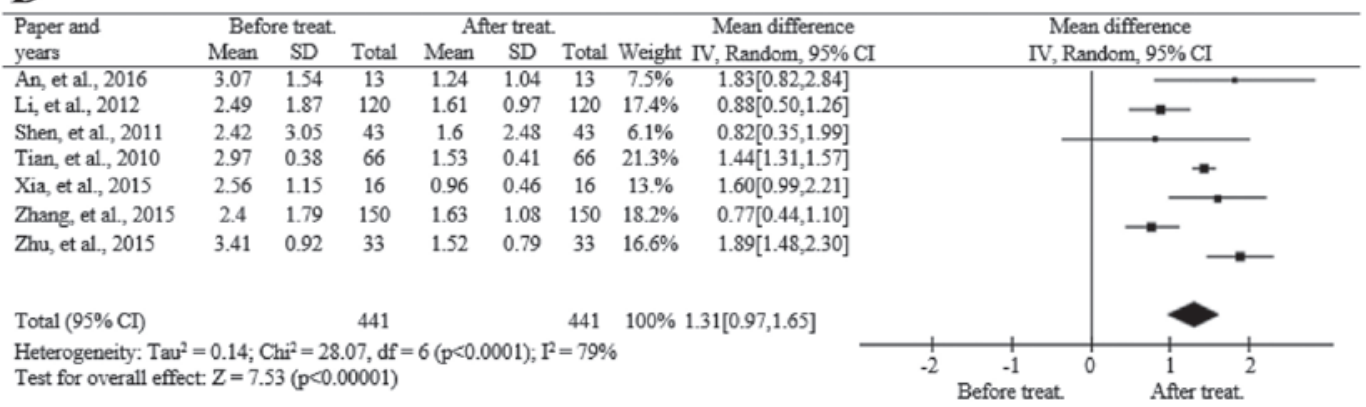

Figure 3. Forest plots for correlation analysis. Forest plots were assessed between (A) healthy controls vs. malignant patients, (B) healthy controls vs. CRC benign patients, (C) benign patients vs. CRC malignant patients and (D) before vs. after surgery. Black diamonds indicate the mean value. CRC, colorectal cancer; SD, standard deviation; CI, confidence interval; IV, inverse variation. 
Meta-analysis random-effects estimates (exponential form)

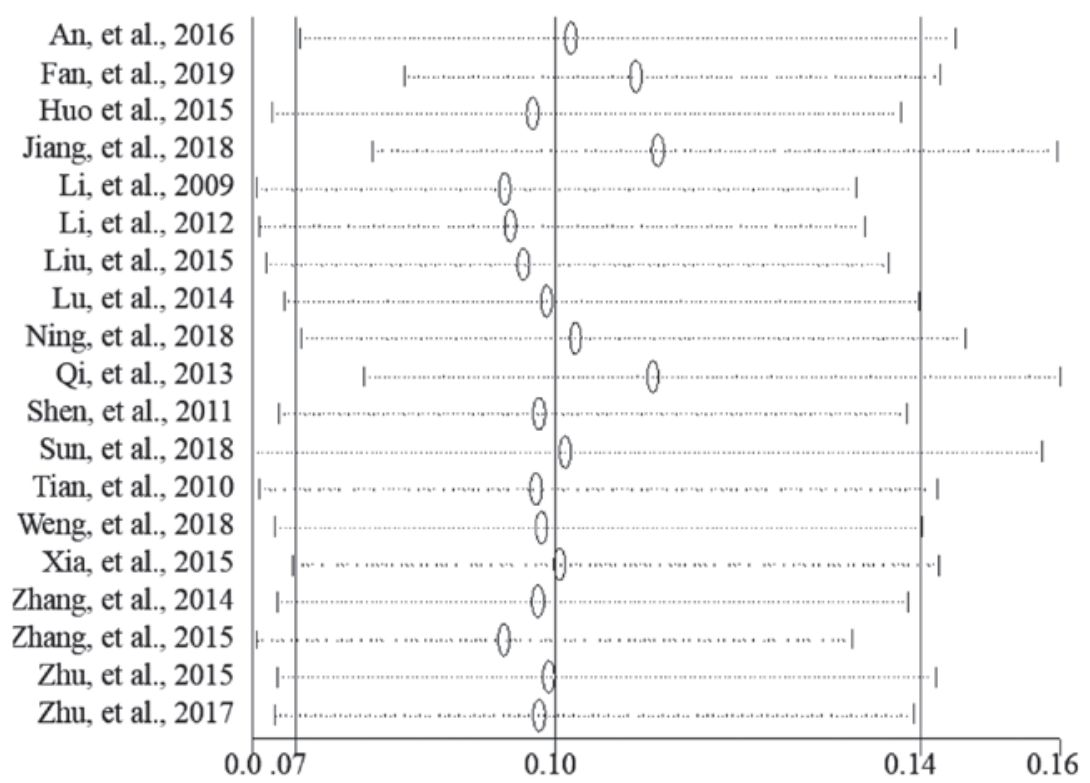

\section{B}

Meta-analysis random-effects estimates (exponential form)

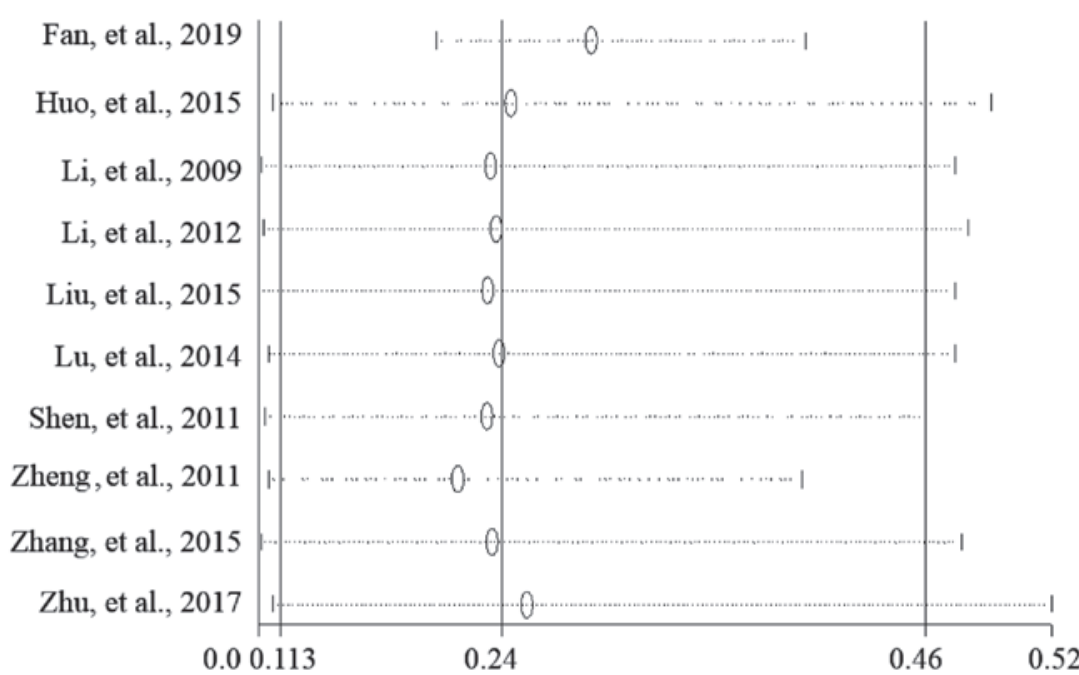

C

Meta-analysis random-effects estimates (exponential form)

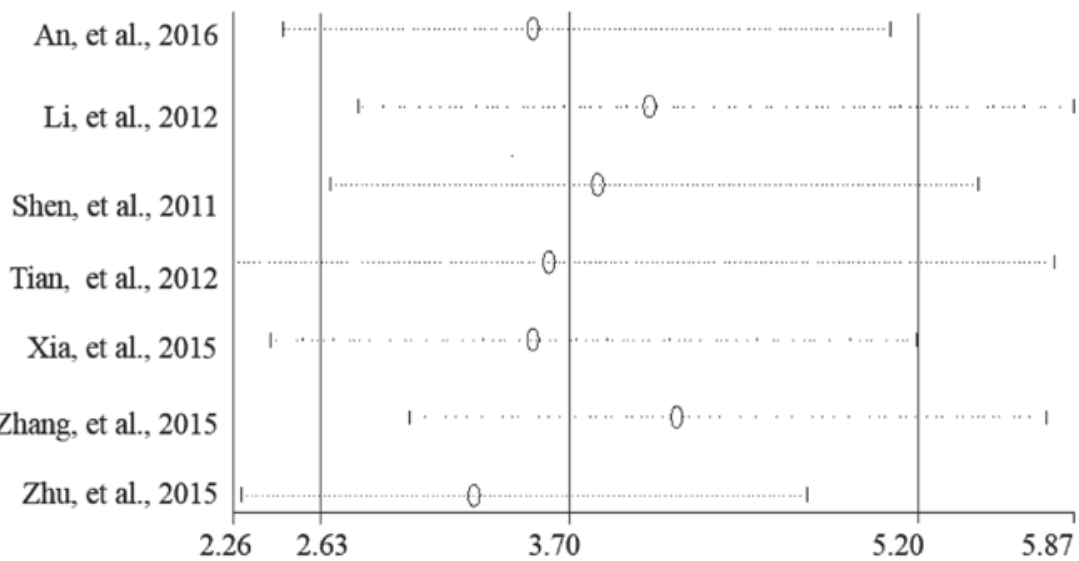

Figure 4. Study sensitivity analyses and the evaluation of mean differences. Evaluations were performed between (A) healthy control group and the colorectal malignancy group, (B) the benign disease and the colorectal malignancy group and (C) between the results obtained before and after surgery in the colorectal cancer group. 
the market today (16). The mean value of STK1p in the benign tumor patients was 1.64-fold higher than that in healthy individuals, but significantly lower than that in CRC patients (1.60-fold). Although the mean STK1p value in benign tumor patients was higher than that in healthy individuals, there was a deviation, with both low and high individual STK1p values. The benign tumor patients with higher STK1p values ( $>2.0 \mathrm{pM})$ may be in a higher risk of developing malignancies than benign tumor patients with low STK1p values $(<2.0 \mathrm{pM})$. Based on a health screening study $(\mathrm{n}=35,365)(18)$, where patients with a STK1p value of $>2.0 \mathrm{pM}$ were found to have a 3-5 times higher risk to develop a malignancy, we concluded that the risk for developing colorectal malignancies from a benign colorectal tumor should be higher in benign tumor patients with an STK1p value of >2.0 pM. On the other hand, benign tumor patients with STK1p values of $<2.0 \mathrm{pM}$ should have a low risk of developing CRC. STK1p was also found to have a prognostic potential in a randomised clinical trial that included patients with CRC $(n=504)$ (28). Patients were followed up for 3-8 years. STK1p was compared with pathological stage and grade, lymph node metastasis, gender and age in relation to survival. A significantly worse survival was found among patients with high STK1p values $(>0.9 \mathrm{pM})$, as compared to patients with low STK1p values $(\leq 0.9 \mathrm{pM})(\mathrm{P}<0.0001)$. Cox regression analysis demonstrated that $\mathrm{STK} 1 \mathrm{p}$, clinical stage and lymph node metastasis were independent prognostic factors, but Dukes' stage $(\mathrm{P}=0.633)$, sex $(\mathrm{P}=0.976)$ and age $(\mathrm{P}=0.520)$ were not.

In the present meta-analysis, the cut-off STK1p value was set to $0.9 \mathrm{pM}$, based on receiver operating characteristic statistical analysis. It is important to understand that the cut-off value of STK1p may differ depending on the type of tumor and how the measurement of STK1p was performed (healthy screening, clinical trials, etc.).

$\mathrm{CRC}$ is a heterogeneous disease, with most cases originating from polyp precursors. Different clinical stages and pathological grades of CRC can lead to heterogeneity.

CRC, a complex disease, is caused by both genetic and environmental factors. Certain studies have shown that inherited genetic factors account for $\sim 35 \%$ of the disease etiology $(53,54)$. It has been suggested that there may be two distinct categories of cancer: Right- and left-sided colon cancers that arise proximally or distally to the splenic flexure, respectively (55). A meta-analysis on vitamin $\mathrm{E}$ concentration in the serum suggested that serum vitamin E concentration was lower in patients with CRC than in healthy controls. Reduced serum vitamin E levels may therefore be a risk factor for CRC. However, prospective cohort studies are still required to assess the risk of serum vitamin $\mathrm{E}$ on $\mathrm{CRC}$ in the future (56). Although the original Dukes staging system has been modified several times, the extent of cancer invasion through the bowel wall and that of regional lymph node invasion is still the mainstay of TNM staging for CRC. A 17,641 patient-cohort study demonstrated that right- and left-sided colon carcinoma (CC) are significantly different in terms of epidemiological, clinical and histological parameters. Right-sided CC has been found to have a worse prognosis. These discrepancies may be due to genetic differences that determine distinct carcinogenesis and biological behaviour (57). There was no significant difference in recurrence rates between right- and left-sided $\mathrm{CC}$ and rectum carcinoma ( $\mathrm{RC})$, but the right-sided CC had a worse prognosis than left-sided $\mathrm{CC}$ and $\mathrm{RC}$, possibly due to more advanced staging and fewer curative resections (58). Another study demonstrated that the patients with stage I right-sided $\mathrm{CC}$ had a significantly better 5 year disease free survival rate than those with left-sided CC; however, no significant difference was observed in the distribution of the first patients with recurrence (59). A retrospective design and single-institution study showed that patients with right-sided colon cancers presented with a significantly increased risk of locoregional recurrence. Right-sided location, female sex, T4 disease, lymph node metastasis, and perineural invasion are independent risk factors for the locoregional recurrence of colon cancer (60). Qin et al (61) found that right- and left-sided colon cancer had significantly different clinicopathological characteristics. Right-sided colon cancer had a higher incidence of recurrence than left-sided colon cancer. Patients with stage III right-sided colon cancer had a worse prognosis than those with stage III left-sided colon cancer. In addition, CRC results may vary from region to region, resulting in heterogeneity, depending on patients' genetic characteristics and living conditions.

With regard to the heterogeneity in the STK1 results, in a preliminary CRC study on 492 patients (Prof. Desong Wan, Sun Yet-Sen University Cancer Centre, China) no significant difference $(\mathrm{P} \approx 0.628-0.645)$ was found in the STK1 values between right- $(\mathrm{n}=91)$ and left- $(\mathrm{n}=124)$ side colon cancer, and rectum $(n=277)$. The STK1p value of right colon, left colon and rectum was $1.8 \pm 1.8,1.9 \pm 1.8$ and $1.9 \pm 1.8$ pM, respectively. STK1p is therefore useful for assessing the proliferation rate in CRC serum samples.

Monitoring the response to treatment is important. Previous studies have shown that STK1p can be used not only to predict prognosis, relapse and survival, but also to monitor tumor treatment (28). In this meta-analysis, the half-life time of STK1p following surgery was found to be $\sim 1$ month, which is the half-life time identified following surgery in patients with lung cancer (26) and gastric carcinoma (62). In the case of gastric carcinoma, the STK1p values were significantly reduced to $52.7 \% 35$ days after open surgery $(\mathrm{P}=0.0106)$. On the contrary, in the patients with distant metastases, the STK1p value increased to $173 \%$ at 35 days post-operatively. There was no significantly difference in TK activity. Similar results were also found in breast cancer (20). However, in the case of minimally invasive surgery, the half-life time of STK1p in patients with bladder carcinoma was only 1 week (63).

It is recommend that STK1p is combined with other tools to evaluate the treatment effect on patients with CRC. This will help individual treatment planning. The following parameters in combination with STK1p should be considered when designing a CRC study: i) CRC is a heterogeneous disease (63), the majority of which is developed from polyp precursors. Therefore, a complete study should use tools useful for the early detection, diagnosis, prognosis and management of CRC development from benign tumors; ii) Since CC and RC are two different types of malignancy (64), they should be evaluated separately; the same goes for rightand left-sided CC and RC; iii) While monitoring the effect of the treatment, the STK1p levels may change depending on clinical stage/grade and tumor type on an individual bases; iv) Since CRC results may differ between living area, depending on the genetic properties and living conditions of the patients, studies should include data from different health centres and oncology hospitals. 
In summary, STK1p could potentially be used for the early detection of benign lesions to prevent their future development into colorectal malignancies, as well as for individual clinical dynamic monitoring of the results of surgery in patients with CRC. The combination of STK1p with colorectal imaging tools after treatment can provide a precise evaluation of the results of the therapy. Together with the use of colorectal-related biomarkers, tumor stage and grade for predicting the risk of relapse, STK1p can help doctors develope more accurate, individualized and rational treatment plans for patients.

\section{Acknowledgements}

The authors would like to thank Professor Desong Wan from the Cancer Centre of Sun Yet-Sen University (Guangdong, China) for providing STK1p serum samples of CRC.

\section{Funding}

No funding was received.

\section{Availability of data and materials}

The datasets used and/or analyzed during the present study are available from the corresponding author on reasonable request.

\section{Authors' contributions}

LD, HM, AH and SX searched the literature, independently extracted the data, produced the figures and tables, and discussed the articles until an agreement was reached. JZ, $\mathrm{EH}$ and SS carefully re-analyzed all results. LD, HM and $\mathrm{AH}$ wrote the first version of the manuscript. EH and SS revised the introduction and discussion sections of the manuscript. All authors read and approved the final manuscript.

\section{Ethics approval and consent to participate}

Not applicable.

\section{Patient consent for publication}

Not applicable.

\section{Competing interests}

JZ is the owner of SSTK Ltd., which produces the STK1p kit. Authors declare they have no competing interests.

\section{References}

1. Boyle P and Levin B: World Cancer Report 2008. International Agency for Research on Cancer; Distributed by WHO Press, c2008, International Agency for Research on Cancer. France, Publisher: Lyon: Geneva, 2008.

2. Stewart BW and Wild CP: World Cancer Report 2014. International Agency for Research on Cancer; World Health Organization. France, Publisher Lyon, 2014.

3. Stewart BW, Bray F, Forman D, Ohgaki H, Straif K, Ullrich A and Wild CP: Cancer prevention as part of precision medicine: 'Plenty to be done'. Carcinogenesis 37: 2-9, 2016.
4. Huxley RR, Ansary-Moghaddam A, Clifton P, Czernichow S, Parr CL and Woodward M: The impact of dietary and lifestyle risk factors on risk of colorectal cancer: A quantitative overview of the epidemiological evidence. Int J Cancer 125: 171-180, 2009.

5. Chen W, Zheng R, Baade PD, Zhang S, Zeng H, Bray F, Jemal A, Yu XQ and He J: Cancer statistics in China, 2015. CA Cancer J Clin 66: 115-132, 2016.

6. Jasperson KW, Tuohy TM, Neklason DW and Burt RW: Hereditary and familial colon cancer. Gastroenterology 138: 2044-2058, 2010.

7. Cappellani A, Zanghì A, D Vita M, Cavallaro A, Piccolo G, Veroux P, Lo Menzo E, Cavallaro V, de Paoli P, Veroux M and Berretta M: Strong correlation between diet and development of colorectal cancer. Front Biosci 18: 190-198, 2013.

8. Abu-Remaileh M, Bender S, Raddatz G, Ansari I, Cohen D, Gutekunst J, Musch T, Linhart H, Breiling A, Pikarsky E, et al: Chronic inflammation induces a novel epigenetic program that is conserved in intestinal adenomas and in colorectal cancer. Cancer Res 75: 2120-2130, 2015.

9. Shussman N and Wexner SD: Colorectal polyps and polyposis syndromes. Gastroenterol Rep 2: 1-15, 2014.

10. Verdecchia A, Francisci S, Brenner H, Gatta G, Micheli A, Mangone L and Kunkler I; EUROCARE-4 Working Group: Recent cancer survival in Europe: A 2000-02 period analysis of EUROCARE-4 data. Lancet Oncol 8: 784-796, 2007.

11. Ciccolallo L, Capocaccia R, Coleman MP, Berrino F, Coebergh JW, Damhuis RA, Faivre J, Martinez-Garcia C, Møller H, Ponz de Leon M, et al: Survival differences between European and US patients with colorectal cancer: Role of stage at diagnosis and surgery. Gut 54: 268-273, 2005.

12. Fearon ER and Vogelstein B. A genetic model for colorectal tumorigenesis. Cell 61: 759-767, 1990.

13. Binefa G, Rodríguez-Moranta F, Teule A and Medina-Hayas M: Colorectal cancer: From prevention to personalized medicine. World J Gastroenterol 20: 6786-6808, 2014.

14. Ribero D, Viganò L, Amisano M and Capussotti L: Prognostic factors after resection of colorectal liver metastases: From morphology to biology. Future Oncol 9: 45-57, 2013.

15. Gasparri F, Wang N, Skog S, Galvani A and Eriksson S: Thymidine kinase 1 expression defines an activated G1 state of the cell cycle as revealed with site-specific antibodies and arrayscan assays. Eur J Cell Biol 88: 779-785, 2009.

16. Zhou J, He E and Skog S: The proliferation marker thymidine kinase 1 in clinical use. Mol Clin Oncol 1: 18-28, 2013.

17. Cao X, Zhou J and Chen Z: Standardized centile curves and reference intervals of serum thymidine kinase 1 levels in a normal Chinese population using the LMS method. Genet Test Mol Biomarkers 20: 445-450, 2016.

18. Chen ZH, Huang SQ, Wang Y, Yang AZ, Wen J, Xu XH, Chen Y, Chen QB, Wang YH, He E, et al: Serological thymidine kinase 1 is a biomarker for early detection of tumours-a health screening study on 35,365 people, using a sensitive chemiluminescent dot blot assay. Sensors (Basel) 11: 11064-11080, 2011.

19. He Q, Zhang P, Zou L, Li H, Wang X, Zhou S, Fornander T and Skog S: Concentration of thymidine kinase 1 in serum (S-TK1) is a more sensitive proliferation marker in human solid tumors than its activity. Oncol Rep 14: 1013-1019, 2005.

20. He Q, Fornander T, Johansson H, Johansson U, Hu GZ, Rutqvist LE and Skog S: Thymidine kinase 1 in serum predicts increased risk of distant or loco-regional recurrence following surgery in patients with early breast cancer. Anticancer Res 26: 4753-4759, 2006.

21. Kumar JK, Aronsson AC, Pilko G, Zupan M, Kumer K, Fabjan T, Osredkar J and Eriksson S: A clinical evaluation of the TK 210 ELISA in sera from breast cancer patients demonstrates high sensitivity and specificity in all stages of disease. Tumour Biol 37: 11937-11945, 2016

22. Liu Y, Ling Y, Qi Q, Tang Y, Xu J, Tong Z, Sheng G, Yang Q and Pan Y: Changes in serum thymidine kinase 1 levels during chemotherapy correlate with objective response in patients with advanced gastric cancer. Exp Ther Med 2: 1177-1181, 2011.

23. Chen F, Tang L, Xia T, He E, Hu G, Zhang M, Li Y, Zhou J, Eriksson S and Skog S: Serum thymidine kinase 1 levels predictcancer-free survival following neoadjuvant, surgical and adjuvant treatment of patients with locally advanced breast cancer. Mol Clin Oncol 1: 894-902, 2013.

24. Aufderklamm S, Hennenlotter J, Todenhoefer T, Gakis G, Schilling D, Vogel U, Kuehs U, Dlugosch J, Knapp J, Merseburger A, et al: XPA-210: A new proliferation marker determines locally advanced prostate cancer and is a predictor of biochemical recurrence. World J Urol 30: 547-552, 2012. 
25. Wang Y, Jiang X, Dong S, Shen J, Yu H, Zhou J, Li J, Ma H, He E and Skog S: Serum TK1 is a more reliable marker than CEA and AFP for cancer screening survey in a study of 56,286 people. Cancer Biomark 16: 529-536, 2016.

26. Lou X, Zhou J, Ma H, Xu S, He E, Skog S and Wang H: The half-life of serum thymidine kinase 1 concentration is an important tool for monitoring surgical response in patients with lung cancer: A meta-analysis. Genet Test Mol Biomarkers 21: 471-478, 2017

27. Yurish SY: Serum-biomarker thymidine kinase 1 for early discovery of tumour process $-160,086$ participants using a sensitive immune-ECL-dot-blot detection system. Advances in Sensors: Reviews 6: 529-540, 2018. http://www.sensorsportal. com.

28. Skog S, He E and Haghdoost S: Prevention and early detection of human tumour. LAP lambert academic publishing, Schaltungsdienst Lange O.H.G., Berlin. p74, p183-257. www. get-morebook.com.

29. Higgins JP, Thompson SG, Deeks JJ and Altman DG: Measuring inconsistency in meta-analyses. BMJ 327: 557-560, 2003.

30. Fan XJ, Shi ZT and Sun W: Significance of TuM2-PK, TK1, CEA, CA19-9 and CA72-4 in the diagnosis of colon cancer patients. Chin J Gerontol 39: 51-53, 2019.

31. Weng YL: Diagnostic value of serum TuM2-PK, TK1 and HER-2 expression levels in rectal cancer. Henan Med Res 1: 1795-1797, 2018.

32. Jiang XL, Bo YX and Ye Y: Correlation between TK1 and tumor markers in diagnosis and pathological features of digestive tract tumors. J Anhui Med Univ 53: 110-114, 2018

33. Sun HW, Mei M, Liu YY and Liu XZ: Clinical value of serum thymidine kinase 1 in the evaluation of chemotherapy for stage IV colon cancer. Continuing Medical Education 8: 81-83, 2018.

34. Ning S, Wei W, Li J, Hou B, Zhong J, Xie Y, Liu H, Mo X, Chen J and Zhang L: Clinical significance and diagnostic capacity of serum TK1, CEA, CA 19-9 and CA 72-4 levels in gastric and colorectal cancer patients. J Cancer 3: 494-501, 2018.

35. Zhu LP, Li XL, Liu H, Ping YF and Zhang YN: Expression and clinical significance of thymidine kinase 1 in rectal cancer. J Xiangnan Univ (Medical Sciences) 19: 9-12, 2017.

36. An N, Zhang M, Lan HT and Li HM: Significance of detecting serum thymidine kinase 1 in metastatic large intestine carcinoma before and after chemotherapy. Med J West China 28: 1257-1260, 2016.

37. Zhu YZ, Ma T,Zhang CJ and Sun GP: The expression and clinical significance of serum thymidine kinase 1 in cancer patients. Acta Univ Med Anhui 7: 1012-1015, 2015.

38. Zeng QH and Zhang SY: Diagnostic value of combined detection of CEA, CA199 and TK1 for colorectal cancer. Med Equipment 10: 2-4, 2015.

39. Xia Y, Liu Y, Qi Q, Zhu M, Zhang Y and Ling Y. Value of serum thymidine kinase 1 in the chemotherapy efficacy evaluation for patients with stage IV colon cancer. J Basic Clin Oncol 28: 6-8, 2015.

40. Liu X, Feng Z, Lai Z, Jiang Y, Xu M and Zhou X: An analysis on expression difference of serum TK1 between patients with gastric carcinoma, lung cancer, colon cancer and healthy person and its clinical significance. J Mathematical Med 58: 913-915, 2015.

41. Huo Y, Yu X and Kong AP: The application value of TK1 in colon cancer patients. Int J Lab Med 36: 3151-3152, 2015.

42. Zhang Y, Wang J, Xie J, Yang D, Han G and Zhang Y: The assay and clinical significance of serum thymidine kinase 1 in patients with colorectal carcinoma. Eur Surg 47: 248-253, 2015.

43. Zhang ZJ, Zheng YP, Lin YH and Mei XQ: Clinical detecting application of TK1 in the diagnosis of common malignant tumors. Int J Lab Med 35:2636-2637, 2014

44. Lu ZQ, Xie YM, Cheng GH and Li JH: Clinical diagnostic value of combined detection of serum TK1, CA199 and CA724 in colorectal cancer. China Practical Medicine 9: 5-7, 2014.

45. Qi QF, Zhu M, Bao YQ and Liu YP: Detection of serum TK1 in patients with colorectal cancer after operation to evaluate the effect of value. World Latest Med Information 13: 60-61, 2013.

46. Li QF: Clinical application of serum thymidine kinase 1 used in colorectal carcinoma patients. Laboratory Med 27: 1080-1081, 2012.

47. Shen J, Chen W, Xu RH, Zhang JF and Jiang H: Evaluation of the significance of serum thymidine kinase 1 detection in the diagnosis and curative effect of gastrointestinal malignant tumors. Exp Lab Med 29: 531-533, 2011.
48. Tian J, Jia Y and Liu H: Serum TK1, EGF, HGF and CRP and IL-6 levels in patiens with colon cancer using laparoscopic and open surgery. Shangdong Med 50: 42-43, 2010.

49. Li X and Wang F: Significance of serum thymidine kinase determination in patients with carcinoma of rectum. Jiangsu Medical J 35: 1436-1437, 2009.

50. Streiner DL: I have the answer, now what's the question? Why meta-analyses do not provide definitive solutions. Can J Psychiat 50: 829-831, 2005.

51. Chatterji M: Grades of evidence: Variability in quality of findings in effectiveness studies of complex field interventions. Am J Eval 28:239-255, 2007

52. Baas M, De Dreu CK and Nijstad BA: A meta-analysis of 25 years of mood-creativity research: Hedonic tone, activation, or regulatory focus? Psychol Bull 134: 779-806, 2008.

53. Zou L, Zhang PG, Zou S, Li Y and He Q: The half-life of cytosolic thymidine kinase in serum by ECL dot bolt potential marker for monitoring the response to surgery of patients with gastric cancer. Int J Biol Markers 17: 135-140, 2002.

54. Zhang J, Jia Q, Zou S, Zhang P, Zhang X, Skog S, Luo P, Zhang W and He Q: Thymidine kinase 1: A proliferation marker for determining prognosis and monitoring the surgical outcome of primary bladder carcinoma patients. Oncol Rep 15: 455-461, 2006.

55. Ogino S, Chan AT, Fuchs CS and Giovannucci E: Molecular pathological epidemiology of colorectal neoplasia: An emerging transdisciplinary and interdisciplinary field. Gut 60: 397-411, 2011.

56. Dong Y, Liu Y, Shu Y, Chen X, Hu J, Zheng R, Ma D, Yang C and Guan X: Link between risk of colorectal cancer and serum vitamin E levels: A meta-analysis of case-control studies. Medicine (Baltimore) 96: e7470 2017.

57. Benedix F, Kube R, Meyer F, Schmidt U, Gastinger I and Lippert H; Colon/Rectum Carcinomas (Primary Tumor) Study Group: Comparison of 17,641 patients with right- and left-sided colon cancer: Differences in epidemiology, perioperative course, histology, and survival. Dis Colon Rectum 53: 57-64, 2010.

58. Lichtenstein P, Holm NV, Verkasalo PK, Iliadou A, Kaprio J, Koskenvuo M, Pukkala E, Skytthe A and Hemminki K: Environmental and heritable factors in the causation of cancer-analyses of cohorts of twins from Sweden, Denmark, and Finland. N Engl J Med 343: 78-85, 2019.

59. Hemminki K and Chen B: Familial risk for colorectal cancers are mainly due to heritable causes. Cancer Epidemiol Biomarkers Prev 13: 1253-1256, 2004

60. Moritani K, Hasegawa H, Okabayashi K, Ishii Y, Endo T and Kitagawa Y: Difference in the recurrence rate between right- and left-sided colon cancer: A 17 year experience at a single institution. Surg Today 44: 1685-1691, 2014.

61. Qin Q, Yang L, Sun YK, Ying JM, Song Y, Zhang W, Wang JW and Zhou AP: Comparison of 627 patients with right- and left-sided colon cancer in China: Differences in clinicopathology, recurrence, and survival. Chronic Dis Transl Med 13: 51-59, 2017.

62. Zou L, Zhang PG, Zou S, Li Y. and He Q: The half-life of cytosolic thymidine kinase in serum by ECL dot blot potential marker for monitoring the response to surgery of patients with gastric cancer. International Journal of Biological Markers 17: 135-140, 2002

63. Zhang J, Jia Q, Zou S, Zhang P, Zhang X, Skog S, Luo P, Zhang $\mathrm{W}$ and $\mathrm{He} \mathrm{Q}$ : Thymidine kinase 1: a proliferation marker for determining prognosis and monitoring the surgical outcome of primary bladder carcinoma patients. Oncology Reports 15: 455-461, 2006.

64. Paschke S, Jafarov S, Staib L, Kreuser ED, Maulbecker-Armstrong C, Roitman M, Holm T, Harris CC, Link KH and Kornmann M: Are colon and rectal cancer two different tumor entities? A proposal to abandon the term colorectal cancer. Int J Mol Sci 19: E2577, 2018.

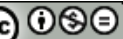

This work is licensed under a Creative Commons Attribution-NonCommercial-NoDerivatives 4.0 International (CC BY-NC-ND 4.0) License. 